\title{
EL GEOÍSMO, UNA PROPUESTA DE ORDENACIÓN TERRITORIAL PLANETARIA
}

\author{
Antonio Lamela \\ Dr. Arquitecto y Presidente Fundador de Estudio Lamela \\ Fernando Moliní Fernández y Juan Vázquez Navarro \\ Departamento de Geografía \\ Universidad Autónoma de Madrid
}

\section{RESUMEN}

El Geoísmo, a través de directrices e indicadores mundiales, pretende contribuir a ordenar territorialmente el Planeta, globalmente, y a través de la planificación supramunicipal, que aplicaría las directrices y se evaluaría respecto a los indicadores. La planificación territorial resulta siempre complicada, incluso en dimensión subnacional, pero, de hecho, aunque sea escasa, ya existe planificación no sólo en ese ámbito, sino incluso en el nacional, y hasta en el continental. La puesta en práctica del Geoísmo no será fácil, pero tal vez no imposible, especialmente si se aplica de manera limitada. Resulta particularmente necesario porque es una respuesta adecuada a la falta de instrumentos con los que la Humanidad debe hacer frente a los avances en el transporte y en las telecomunicaciones, así como a la creciente e imparable globalización.

Palabras clave: Geoísmo, ordenación del territorio, planificación territorial, planificación supramunicipal, Estrategia Territorial Europea.

\section{ABSTRACT}

Geoism, through world directives and indicators, aims to contribute towards the organisation of territory, firstly of the planet as a whole in response to global problems and secondly, of parts of the planet, through supra-local planning, applying these directives and evaluating it according to the indicators. Territorial planning is always complicated even on a sub-national dimension. However, although it may be scarce, there is planning not only on this scale, but also nationally and even at a continental level. It's difficult to put Geoism into practice, but not impossible, particularly if applied in a limited fashion. It is especially necessary because it is a suitable response to the lack of tools with which humanity deals with advances in transportation and telecommunications, as well as with increasing globalisation.

Key words: Geoism, territorial planning, supra-municipal planning, European Spatial Strategy. 


\section{El Geoísmo}

El Dr. arquitecto Antonio Lamela planteó desde muy temprano (Lamela, 1976) la necesidad de una ordenación del territorio que considerase desde el ámbito planetario hasta el supramunicipal. A esta idea, que ha seguido defendiendo hasta la actualidad (Lamela, 2003), la denominó Geoísmo y se puede definir como una propuesta de disciplina y de práctica profesional que pretende contribuir a ordenar territorialmente, en primer lugar, el Planeta en su conjunto, dando respuesta a los problemas globales, por ejemplo, a través de directrices e indicadores mundiales y, en segundo lugar a partes del mismo, mediante la planificación supramunicipal, que tendría en cuenta aquellas directrices, y que se evaluaría respecto a tales indicadores.

El Geoísmo guarda cierta relación con otros trabajos contemporáneos, aunque también presentan notables diferencias. Así, en el primer informe al Club de Roma, publicado en 1972 bajo el título «Los límites del crecimiento», es posible leer una escueta referencia a la necesidad de una planificación que, aunque no lo diga explícitamente, se puede entender que sería mundial. Sus autores escriben: «no dudamos que si la Humanidad ha de embarcarse en una nueva vía, antes será necesario concertar medidas internacionales y realizar una planeación — sic - conjunta de largo alcance en una escala y amplitud sin precedentes» (Meadows y otros, 1972: 242). Hablan de planificación, pero en ningún momento hacen referencia a la perspectiva territorial. Dos años más tarde, la misma institución recibió un segundo informe titulado La Humanidad en la Encrucijada. En él se establece una analogía entre la Naturaleza y el Sistema Mundial, en la que se menciona de manera puntual la conveniencia de un plan mundial, sin aludir tampoco a sus aspectos territoriales (Mesarovic y Pestel, 1975: 28 y 186). Recientemente, Doval (2004: 187) ha señalado la necesidad de «planificar globalmente el espacio geográfico mundial para acometer la resolución de los grandes problemas globales de naturaleza física y humana que hoy tiene planteados la humanidad en su conjunto».

El objetivo de este artículo es avanzar en cómo esto puede ser posible. Intenta ser algo más práctico que las obras anteriormente mencionadas, que se centran en explicar la necesidad de una planificación mundial sin entrar en propuestas de cómo se podría realizar. Asimismo, se exponen algunos precedentes que muestran los tímidos avances que se han realizado en esa dirección, si bien se reconoce la enorme magnitud de los obstáculos a los que se enfrenta.

Una ordenación territorial planetaria es una tarea extraordinariamente difícil, pero tal vez no imposible, como se intenta argumentar en este artículo. En cualquier caso, no se propugna un tipo de plan formal y rígido, sino unas directrices territoriales flexibles, que se pudiesen introducir de manera parcial, y que un número significativo de naciones pudiesen adoptar por consenso.

\section{Planificación territorial nacional en modelos descentralizados}

Hay casos que indican que puede haber una planificación territorial de ámbito nacional en países altamente federalizados, como Suiza. Pujadas y Font (1998: 196) destacan que Suiza es una nación que logra una considerable coordinación federal de sus planes regionales. Afirman: «cada Cantón tiene competencias plenas en ordenación del territorio y elabora su propio Plan Director Cantonal, pero la necesidad de reforzar la coordinación por parte del gobierno federal ha llevado a la fórmula de las «concepciones», entendidas como un sistema normativo de objetivos y medidas que por lo general no tiene carácter vinculante, pero constituyen puntos de referencia importantes para la elaboración de los 
planes cantonales. En 1995 el gobierno federal presentó un documento denominado Bases para la ordenación del territorio suizo, que aportaba, tanto a las administraciones públicas como al sector privado, un marco orientativo para la toma de decisiones con incidencia territorial».

En Suiza, el planeamiento territorial es principalmente responsabilidad de los cantones, aunque el gobierno nacional tiene ciertas competencias formales, que están reflejadas en la Ley Federal de Planificación Territorial de 1979. La Asociación Suiza de Planificación (VLP-ASPAN, 2004: 3) señala que el principal objetivo de esta ley es optimizar la utilización de su limitado territorio, algo imprescindible, porque únicamente el treinta por ciento resulta adecuado para un uso humano intensivo.

El sistema de planificación territorial suizo ha sido descrito recientemente por un informe de la OCDE (OECD, 2002: 12-14 y 86-97). Considera a Suiza la nación más descentralizada de cuantas componen esta organización, particularmente por la competencia fiscal de sus cantones. El funcionamiento resulta razonablemente bueno, ya que valores como la coordinación y la cooperación son asumidos en la práctica por todas las administraciones, si bien hay una creciente tensión entre la autonomía local, la cohesión nacional y la necesidad de una mayor colaboración entre los cantones. La Oficina Federal de Planificación Espacial tiene la responsabilidad de coordinar y aprobar el planeamiento territorial cantonal y de establecer los objetivos de la planificación regional y municipal, pero, fundamentalmente, sus tareas se limitan a realizar estudios y a establecer los principios generales, estando la estrategia económica territorial fuera de su alcance. Sin embargo, como ya se ha comentado, los cantones pueden desarrollar políticas económicas y fiscales, por ejemplo, para atraer actividad económica o población.

En 1996 se publicaron las mencionadas Bases para la ordenación del territorio suizo. El documento muestra una visión territorial a largo plazo de la nación, y expone los principales retos para lograr un uso más eficaz y más equilibrado del espacio. Suiza presenta un territorio montañoso, gran abundancia de áreas con valor natural, paisajes con un importante atractivo turístico, tamaño reducido, densidad relativamente alta, una pujanza económica notable, y escasez de terrenos adecuados para nuevos desarrollos. Esto hace que todos sus niveles administrativos concedan una gran atención a la ordenación del territorio. Y explica que una de las principales preocupaciones de la planificación sea la optimización de la organización espacial, lo que lleva a proponer un uso intensivo del territorio disponible, que, entre otras medidas, implica una concentración descentralizada de núcleos compactos de diferentes tamaños, dispuestos en red, así como detener la expansión desordenada de viviendas unifamiliares. Sin embargo, su aplicación no parece tener mucho éxito en lo que respecta a lograr unas densidades más elevadas, porque, según Luzón (2004: 708) «la tendencia es mantener o incluso rebajar la densidad de población en muchos municipios».

Para reflexionar sobre la viabilidad de una planificación territorial integral de ámbito nacional en contextos descentralizados, otro caso, aún más interesante, es el de Austria, porque lo ha logrado elaborar, a pesar de que el gobierno federal carece de competencias formales en la materia. Esta falta de capacidad teórica contrasta con el hecho de que el Estado central es responsable de la mayoría de las inversiones con incidencia territorial. Faludi (1998) describe el funcionamiento del sistema austriaco, y lo pone como ejemplo de que los acuerdos informales pueden funcionar. La planificación la elabora la Conferencia Austriaca de Planificación Espacial, creada en 1971. Dicha Conferencia está formada por representantes federales, estatales y municipales, además de contar con apoyo técnico estable, cuya continuidad no está sujeta a los resultados electorales. Funciona con éxito, a pesar de tener un carácter voluntario, porque todas las partes perciben que hay una necesidad importante de coordinación y de cooperación. El principal limitante de este modelo 
deriva de que las decisiones, al ser tomadas por unanimidad, únicamente representan el mínimo común denominador al que están dispuestas a llegar las partes. Aún así, las recomendaciones que se aprueban pueden tener un gran valor, como ha ocurrido con las Perspectivas Austriacas de Planificación Territorial, la primera de ellas aprobada en 1981. Esta ordenación territorial ha resultado muy positiva en muchos sentidos. Entre otros logros, cabe destacar haber permitido alcanzar un consenso básico de política espacial, mejorar la coordinación, comprender las diferencias entre las distintas administraciones, establecer un proceso de aprendizaje mutuo, crear una cultura espacial, mostrar que la cooperación voluntaria es posible, etc.

\section{Un caso de planificación continental: la Estrategia Territorial Europea}

Posiblemente, la ordenación territorial realizada hasta la fecha que abarque a un mayor número de naciones es la Estrategia Territorial Europea (ETE), subtitulada Hacia un desarrollo equilibrado y sostenible del territorio de la Unión Europea. Tardó diez años en ser elaborada. Comenzó en una reunión informal de los Ministros responsables de ordenación del territorio en Nantes, en 1989, y culminó con la aprobación de la ETE en una reunión de las mismas características en Potsdam, en 1999, en la que participó también el responsable de política regional de la Comisión Europea. Por primera vez se pretendió observar la situación global del territorio europeo y superar la perspectiva parcial que hasta entonces ofrecían las políticas sectoriales, así como presentar una visión futura del territorio que sirviera de marco general de referencia.

La ETE se planteó tres objetivos fundamentales (CE, 1999: 11): cohesión económica y social; conservación de los recursos naturales y del patrimonio cultural; y competitividad más equilibrada del territorio europeo. Pretendía promover el desarrollo sostenible mediante una estructura equilibrada del territorio, para lo que establece tres directrices de política de desarrollo territorial: el desarrollo de un sistema equilibrado y policéntrico de ciudades y una nueva relación entre campo y ciudad; la garantía de un acceso equivalente -en el sentido de paritario, según el término utilizado en inglés - a las infraestructuras y al conocimiento; y el desarrollo sostenible, la gestión inteligente y la protección de la Naturaleza y del patrimonio cultural. Algunas de las directrices son discutibles. Por ejemplo, parece imposible el acceso equivalente a las infraestructuras; a lo sumo, se podría intentar establecer un acceso mínimo a ellas ${ }^{1}$.

El que se hayan formulado unos principios territoriales rectores ya representa un gran avance, aunque sólo fuesen el comienzo de la descripción de lo que se pretende alcanzar. Éstos definen algunas posiciones de interés. Por ejemplo, dejan claro que no se opta por una jerarquía bien definida de ciudades, que tenga un gran centro dominante, sino por un sistema múltiple que contribuya a evitar la excesiva concentración de riqueza y población en el núcleo central de la Unión Europea. No explican suficientemente cómo debe ser el modelo policéntrico que propugnan, pero si describen algunos de sus elementos: «la creación de varias zonas dinámicas de integración en la economía mundial, bien distribuidas por la UE y formadas por regiones metropolitanas de fácil acceso internacional, enlazadas entre sí y bien conectadas con las regiones rurales y las ciudades, de distinto tamaño, de sus áreas de influencia, desempeñará un papel clave en la mejora del equilibrio territorial de Europa» (CE, 1999: 22).

1 Las críticas que se podrían hacer a la ETE estarían ocasionadas, en parte, porque la traducción del inglés, a veces, parece poco afortunada. Así, se traduce «prudent management» por gestión inteligente, en vez de por gestión prudente, haciendo que la directriz pierda coherencia. 
Faludi (2004a: 395) ha criticado que la ETE se haya abstenido de visualizar la estructura espacial que se proponía para Europa. Lo considera un problema importante: «lo que llama la atención es la ausencia de cualquier tipo de esquema o plan general que sirva para conceptualizar el espacio europeo. Esto es algo muy serio. Al fin y al cabo, de lo que se trata es de conceptualizar el territorio de la UE y los efectos que este tiene sobre - entre otras cosas - la competitividad europea. Resulta difícil concebir que esto se lleve a cabo sin ningún tipo de representación del territorio afectado» (2004b: 20-21).

La ETE no es jurídicamente vinculante, pero a pesar de ello ha pretendido constituir un marco adecuado de orientación y de cooperación para las políticas sectoriales con repercusiones territoriales de la Comunidad, los Estados miembros y las autoridades regionales y locales (CE, 1999: 12). La valoración de su éxito está sujeta a interpretación. Depende, sobre todo, de que se espere mucho o poco de ella, por lo que, a veces, la posición de los autores podría incluso parecer contradictoria. Andreas Faludi tiene comentarios desfavorables y favorables, aunque da primacía a los segundos. Entre los primeros menciona que «tan pronto como la ETE estuvo impresa, la Comisión dejó de apoyarla» y que «ha sido dejada de lado por la cuestión de dónde reside la competencia de la planificación» (Faludi, 2004c: 1350 y 1361). Asimismo, señala que se necesitaría un esfuerzo hercúleo para resucitar el proceso de la ETE (Faludi, 2004a: 404), y que el talón de Aquiles de la política europea de ordenación del territorio es la falta de coordinación continua entre las Direcciones Generales (Faludi, 2004b: 39). A pesar de ello, su valoración general es favorable. Afirma que «los Estados miembros y la Comisión están ahora aplicando la ETE» (Faludi, 2004b: 23) y a la misma conclusión llega en un estudio más detallado de algunas naciones (Faludi, 2004a: 395). Globalmente piensa que la ETE se puede considerar un éxito, porque sus conceptos se han incorporado en varias políticas o juegan un papel en la planificación, debido a la difusión de los mismos (Idem: 405).

La Comisión Europea adopta muchas de las ideas de la ETE a través de su política de cohesión territorial, respecto a la que el proyecto de Constitución de la Unión Europea le reconoce competencia compartida con los Estados miembros (artículo I-14.2.c). El último documento europeo relacionado con la materia es el denominado tercer informe sobre la cohesión económica y social (EC, 2004). Aunque en la portada no aparezca la mención expresa a la cohesión territorial, el documento tiene una gran dimensión espacial, si bien no agota todos los aspectos de la ETE, que convendría que fuese revitalizada en si misma.

\section{Medidas parciales planetarias: los convenios con incidencia territorial}

No existe una planificación territorial a escala planetaria, aunque de manera progresiva se va disponiendo de un número cada vez mayor de tratados y protocolos mundiales con repercusiones territoriales. La tabla 1 resume los más importantes.

No se han incluido ni las declaraciones ni los acuerdos que no sean convenios. Por ejemplo, no se reflejan las correspondientes a la conferencia mundial de Río de Janeiro de las Naciones Unidas sobre Medio Ambiente y Desarrollo — 1992_, a pesar de que, además de los tratados, se han generado otros procesos de gran interés, como ocurre con las Agendas 21 Locales, que se derivan del Programa 21, que forma parte de esta conferencia (DGCEA, 1998). Así, quedan también fuera otros acuerdos de gran trascendencia, como los que protagoniza la Organización Mundial del Comercio, dedicada a establecer normas negociadas que rigen el comercio entre los países a nivel mundial o casi mundial.

El número de tratados y las cuestiones abordadas evidencian que se ha avanzado mucho, pero se carece de una estrategia general que coordine e integre las temáticas abordadas, es decir, faltan las funciones propias de la planificación territorial. No se dispone de una 
Tabla 1

PRINCIPALES TRATADOS MUNDIALES CON UNA MAYOR INCIDENCIA TERRITORIAL

\begin{tabular}{|c|c|}
\hline Convenio de Chicago sobre aviación civil internacional & 1944 \\
\hline Convenio de Washington D.C. para la regulación de la caza de ballenas & 1946 \\
\hline Tratado del Antártico, de Washington & 1959 \\
\hline $\begin{array}{l}\text { Tratado de Moscú, sobre prohibición de pruebas con armas nucleares en la atmósfera, en el espacio } \\
\text { ultraterrestre y bajo el agua }\end{array}$ & 1963 \\
\hline $\begin{array}{l}\text { Tratado sobre los principios que deben regir las actividades de los Estados en la exploración y utilización del } \\
\text { espacio ultraterrestre, incluso la Luna y otros cuerpos celestes }\end{array}$ & 1967 \\
\hline Tratado de Londres, Moscú y Washington sobre la no proliferación de armas nucleares & 1968 \\
\hline Convenio de Viena sobre el derecho de los tratados & 1969 \\
\hline $\begin{array}{l}\text { Convención de Ramsar relativa a los humedales de importancia mundial, particularmente como hábitat de } \\
\text { aves acuáticas }\end{array}$ & 1971 \\
\hline Convenio de Londres sobre prevención de la contaminación del mar por vertido de desechos y otras materias & 1972 \\
\hline Convención de París para la protección del patrimonio mundial cultural y natural & 1972 \\
\hline Convenio de Londres sobre prevención de la contaminación por buques & 1973 \\
\hline $\begin{array}{l}\text { Convenio de Washington sobre el comercio internacional de especies amenazadas de la fauna y flora } \\
\text { silvestres }\end{array}$ & 1973 \\
\hline Convenio de Barcelona de protección del mar Mediterráneo contra la contaminación & 1976 \\
\hline Convención de Ginebra sobre la contaminación atmosférica transfronteriza de largo alcance & 1979 \\
\hline Convención de Bonn sobre la conservación de las especies migratorias de animales silvestres & 1979 \\
\hline Convención de Montego Bay sobre el Derecho del Mar & 1982 \\
\hline Convenio de Viena para la protección de la capa de ozono & 1985 \\
\hline $\begin{array}{l}\text { Convenio de Basilea sobre el control de los movimientos transfronterizos de los desechos peligrosos y su } \\
\text { eliminación }\end{array}$ & 1989 \\
\hline Convenio de Londres sobre cooperación, preparación y lucha contra la contaminación por hidrocarburos & 1990 \\
\hline $\begin{array}{l}\text { Convenio de Helsinki sobre protección y uso de los cursos de agua transfronterizos y de los lagos } \\
\text { internacionales }\end{array}$ & 1992 \\
\hline Convenio de Río de Janeiro sobre la diversidad biológica & 1992 \\
\hline Convenio marco de Nueva York sobre el cambio climático & 1992 \\
\hline $\begin{array}{l}\text { Convención de París contra la desertización en los países afectados por sequía grave o desertificación, en } \\
\text { particular en África }\end{array}$ & 1994 \\
\hline Convención de Nueva York sobre el derecho de los usos de agua para fines distintos de la navegación & 1997 \\
\hline Protocolo de Kioto de la convención marco sobre cambio climático & 1997 \\
\hline Protocolo de Gotemburgo para reducir la acidificación, la eutrofización y el ozono troposférico & 1999 \\
\hline $\begin{array}{l}\text { Protocolo de Kiev de responsabilidad civil y compensación por el daño causado por el efecto en aguas } \\
\text { transfronterizas de accidentes industriales }\end{array}$ & 2003 \\
\hline
\end{tabular}

Fuente: elaboración propia basada en Delgado y otros (eds.), 2004; González y Sáenz, 2003; y diversas páginas en la Red, entre ellas UNEP, 2005; SEDAC, 2005 y ONU, 2005. 


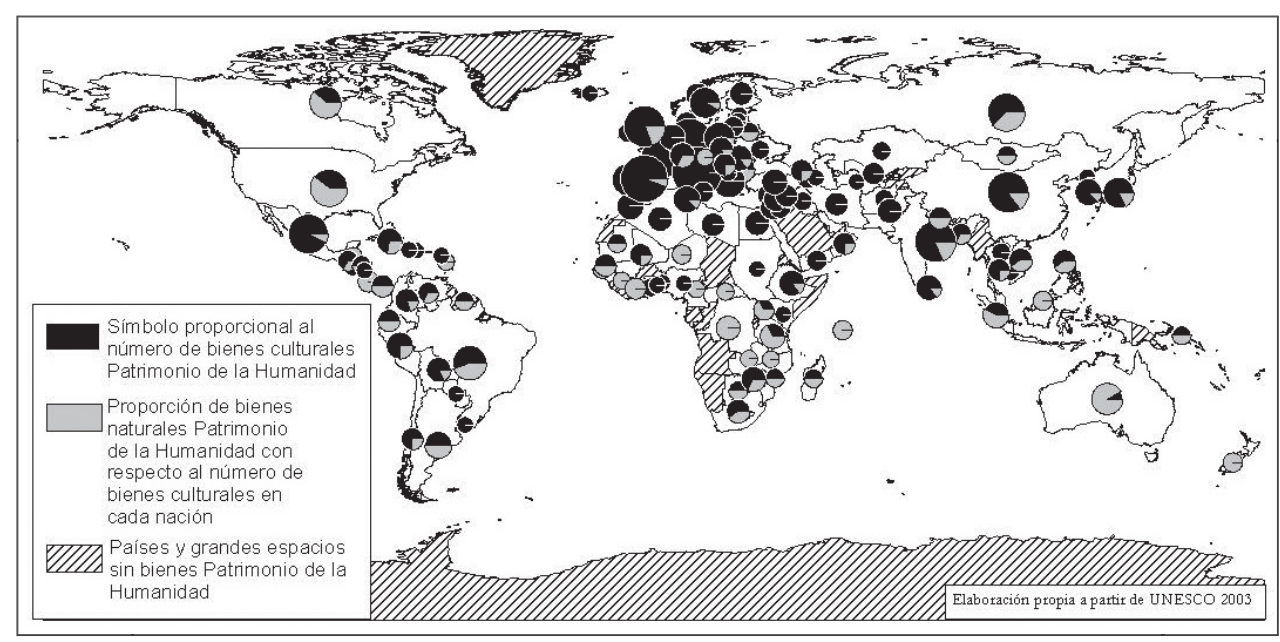

FIgURa 1. Lugares declarados Patrimonio de la Humanidad

visión de conjunto que muestre lo ya realizado y que permita decidir lo que se debería hacer de manera más inmediata por resultar prioritario. Además, se omiten aspectos territoriales importantes, como, por ejemplo, regular los tipos de asentamientos humanos que resulten más sostenibles ${ }^{2}$.

Lógicamente, la aplicación de las medidas mundiales no se realiza de manera uniforme. A pesar de tener un alcance global, se suelen concretar más en determinados tipos de naciones, en función de su desarrollo económico, su historia y su geografía. La figura 1 muestra los sitios declarados Patrimonio de la Humanidad, englobando tanto los culturales como los naturales y los mixtos. Se observa su presencia en casi todas las naciones del mundo, a la vez que una marcada concentración en Europa.

El que una actuación incida principalmente en un determinado conjunto de países no significa necesariamente que tenga una menor utilidad mundial o que genere desigualdades injustificadas. Es bueno que algunos lugares sean pioneros, en espera de que otros potencialmente implicados tengan la disposición de seguirlos y estén en condiciones de hacerlo.

Las medidas parciales planetarias que se están adoptando son muy meritorias, pero no dejan de ser insuficientes. Dos muestras serían:

- El Protocolo de Kioto - 1997— no ha sido aún ratificado por la nación más contaminante, los EE UU, en gran medida por la presión, en contra, que ejerce su propia industria. A su vez, en las naciones que lo pretenden hacer cumplir hay numerosas empresas que amenazan con trasladarse a otros países más permisivos. Además de por los intereses de la industria, su aplicación también falla porque: no se realiza a escala planetaria; su objetivo ${ }^{3}$ y posibles logros son muy modestos respecto al problema planteado; y, tal vez, habría otras alternativas mejores a las que dedicar el coste que implica, como el fomentar la investigación en energía solar, así como en la captura de $\mathrm{CO}_{2}$ y otros contaminantes.

2 Sí ha habido diversas conferencias de las Naciones Unidas sobre asentamientos humanos, con medidas de gran interés como el Programa Hábitat y los catálogos de buenas prácticas.

3 Reducir, para el año 2012, las emisiones de los seis gases de efecto invernadero de los países desarrollados que lo han ratificado, en un 5,2\% de media en el periodo 2008-2012, respecto al nivel de 1990. 
- La Agenda 21 - 1993 - se extiende muy lentamente. Riego (2004: 105) comenta: «en todo el mundo no se han llegado a implantar más allá de 7.000 Agendas 21 Locales ... A este ritmo de 7.000 Agendas 21 Locales en 11 años, harían falta más de 1.500 años (ii15 siglos!!) para llevar a cabo la implantación de las Agendas 21 Locales en ese más de un millón de municipios que tiene el Planeta». Podría ocurrir que, en un momento dado, el ritmo de creación de Agendas 21 se acelerase, pero de momento la tendencia es demasiado pausada.

\section{Organización Mundial de la Ordenación del Territorio}

El Geoísmo se podría instituir mediante la creación de una Organización Mundial de la Ordenación del Territorio - OMOT_, que reuniese a los ministros relacionados con el tema de todas las naciones interesadas. Debería contar con una Secretaría que les prestase un apoyo técnico permanente y con un Consejo Consultivo al más alto nivel, en el que estuviesen representados los agentes sociales: sindicatos, organizaciones ecologistas, organizaciones no gubernamentales, empresarios, etc. La OMOT debería intentar analizar lo más objetivamente posible los problemas territoriales, proponer medidas que mejoren las situaciones y avanzar hacia una gobernabilidad mundial más racional, en la que participen, en condiciones de mayor igualdad, los diferentes agentes humanos involucrados, si bien, la capacidad de decisión final la ostentan los Estados. Se debe propugnar llegar a actuaciones de consenso, que, al menos, reflejen el mínimo común denominador que puedan acordar las diferentes partes afectadas. En numerosas cuestiones fundamentales será imposible llegar a acuerdos unánimes, como ha sucedido con el Protocolo de Kioto, pero, aunque únicamente se logren ciertas mejoras, siempre será un avance. Asimismo, se debe intentar ofrecer una visión esperanzadora sobre el modelo territorial futuro, ilusionante, que marque una dirección preferente hacia dónde movilizar el mayor número posible de esfuerzos.

Para poder financiar las medidas que se decidan adoptar, se podría contar con un fondo geoísta, nutrido, entre otras fuentes, por los países desarrollados que todavía no alcanzan el 0,7\% del PIB. en ayuda al desarrollo, por ejemplo, en función de las aglomeraciones urbanas que tengan más de un cierto número de habitantes y que estén creciendo. Hay que seleccionar un umbral a partir del cual se podría considerar que empiezan a ser conjuntos urbanos algo más globales, y que, por tanto, se deberían preocupar más intensamente por los asuntos mundiales ${ }^{4}$. Por el contrario, las ciudades de menor tamaño se supone que, comparativamente, podrían tender a preocuparse más por los asuntos locales y rurales. Además, las ciudades de tamaño mediano y grande tienen mayores economías de escala y de especialización, por lo que se les supone una mayor capacidad para competir globalmente. Los recursos obtenidos por el fondo geoísta servirían para financiar actuaciones clave desde un punto de vista territorial, como proyectos de ecodesarrollo para proteger los espacios naturales más valiosos y su entorno, o la creación de nuevos barrios razonablemente densos y con abundantes espacios verdes en las ciudades de los países en vías de desarrollo, que contribuyan a absorber los excedentes demográficos producidos por la liberalización y la modernización agrícola. En la figura 2 se han reflejado las ciudades de más de 500.000 de habitantes y las selvas tropicales, resaltando algunos de los elementos básicos de la propuesta.

4 El umbral se debería precisar con estudios detallados, que contemplen cual es el resultado de su aplicación. Por ejemplo, se podría establecer una cifra de 200.000 habitantes, porque, si fuese mayor, dependiendo de cómo se mida, podrían quedar fuera del cómputo estructuras polinucleares de ciudades de tamaño pequeño o mediano de países desarrollados. Esto podría sucederle a todos los núcleos urbanos de Suiza si, por ejemplo, se adoptase la cifra de 500.000 de habitantes. 


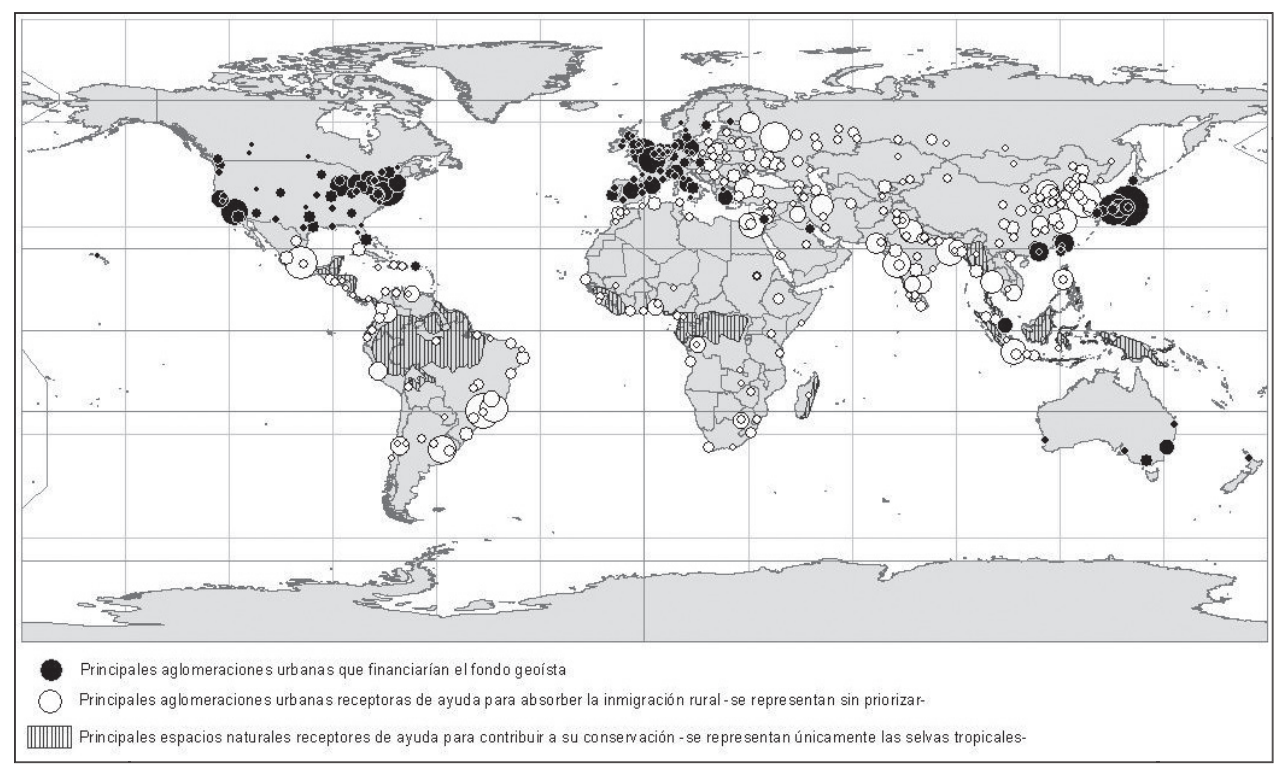

FIGURA 2. Principales lugares donantes y receptores de un fondo geoísta.

Fuente: elaboración propia a partir de Banco Mundial (2003: 251) y UNEP (2005).

La OMOT intentaría actuar como contrapeso de la Organización Mundial del Comercio - OMC_-, cuyas políticas tienden a ignorar las consecuencias territoriales que implican. En materias diferentes al comercio, como las cuestiones ambientales, «su única tarea es estudiar los problemas que surgen cuando las políticas de medio ambiente tienen efectos importantes en el comercio» (OMC, 2003: 65). Así, la desregulación de la agricultura conlleva problemas ecológicos y sociales, a los que en la actualidad no se les ofrece respuesta. Entre los primeros cabe destacar que la creciente liberalización expande las oportunidades agrícolas de los países en vías de desarrollo y, con ello, incita a la roturación de sus espacios naturales (Barbier, 2000; Rudel, 2002; Hecht, 2005). Asimismo, contribuye a difundir la agricultura industrializada, con los problemas medioambientales que genera, como el incremento del consumo de energía no renovable, el deterioro de suelo, la contaminación del agua, la pérdida de diversidad genética, etc. (Horrigan y otros, 2002; Ehrenfeld, 2005). Respecto a lo problemas sociales, Amin (2004: 131-132) calcula que, de los aproximadamente 3.000 millones de labradores del Tercer Mundo, se podría pasar a unos 20 millones de granjeros modernizados. El resto, en su inmensa mayoría, se empobrecería todavía más respecto a las condiciones de miseria en que ahora vive, porque incluso en la hipótesis poco realista de un crecimiento industrial del $7 \%$ anual, no se absorbería ni tan siquiera a un tercio de los trabajadores desplazados.

Se puede opinar que el Geoísmo es utópico, al pretender instituciones que funcionen mediante consenso en un mundo con aproximadamente 200 naciones. Efectivamente, su consecución parece altamente difícil, pero se pueden esgrimir diversos argumentos a favor de que no es totalmente imposible, entre los que destacan:

- La mundialización, en cierta medida, fuerza a lograr el consenso, porque puede paliar en parte el proceso de la creciente pérdida de las soberanías nacionales. Los 
países se ven obligadas a entenderse porque, si no alcanzan mínimos comunes denominadores, probablemente tendrán un menor control sobre su propio destino.

- El que las empresas simultaneen la cooperación y la competencia se ha descrito como una estrategia altamente eficaz, incluso la mejor según algunos autores. Nalebuff y Brandenbuerguer (1997: 259) han sido pioneros en teorizar las ventajas que adquieren las empresas que saben combinar, por una parte, la creación de valor de manera cooperativa, incrementando el resultado a repartir mediante la ayuda mutua, con, por otra, su apropiación de manera competitiva. Si esto fuese cierto para las sociedades mercantiles, se puede suponer que tanto más lo sería para las naciones.

- Austria, un país federal cuyo gobierno central carece de competencias en ordenación del territorio, ha mostrado que se puede hacer una planificación territorial del conjunto de la nación basada en acuerdos de carácter voluntario.

- Hay instituciones con una gran influencia mundial que prácticamente siempre han funcionado por consenso. Este es el caso de la $\mathrm{OMC}$, que en la actualidad integra a 148 naciones que representan la casi la totalidad del comercio mundial. A la OMC le sería posible adoptar decisiones mayoritarias, pero esta opción nunca la ha utili$z^{2} a^{5}$. Sus decisiones se adoptan por consenso y se ratifican por el parlamento de todos los estados miembros, aplicándose por igual tanto a las naciones ricas como a las pobres (Peet, 2004: 199-200). Lógicamente, este procedimiento de toma de decisiones no significa que todas ellas hayan sido acertadas, particularmente cuando se han opuesto los intereses comerciales a los medioambientales.

- Aunque finalmente no se lograse ningún tipo de consenso, el debate que se produciría ya sería por sí mismo de gran utilidad.

\section{Planificación geoística}

Los principales elementos de la planificación geoística consistirían en la elaboración de unas directrices territoriales sobre las medidas más eficaces que se pueden adoptar a escala mundial y en la selección de unos indicadores que sirvan para medir la eficacia de las propuestas planteadas, según se recoge en la figura 3.

Los objetivos no deben ser simplemente genéricos, sino que conviene concretarlos. Un buen punto de partida son los Objetivos de Desarrollo del Milenio, vinculados a la Declaración del Milenio de las Naciones Unidas, que fue subscrita en el año 2000 «por la mayor concentración de jefes de Estado de la Historia» (Fukuda-Parr, 2003: 1). Habría que completar los Objetivos de Desarrollo del Milenio con otros de carácter más territorial, por ejemplo, referidos a la protección de espacios con valor ecológico, la gestión de recursos naturales, la distribución de los asentamientos urbanos, las redes de transporte, etc., así como contribuir a su consecución mediante una visión más completa e interrelacionada, que busque sinergias y evite disfunciones.

Las directrices territoriales deben tender a ser integrales, pero pueden empezar centrándose en los aspectos más relevantes de la planificación territorial mundial, aunque sea de forma individualizada. Un ejemplo de directriz se podría referir al precio del agua, cuestión en la que autores muy dispares coinciden en considerar clave. Brown (2004: 172-173) estima que la Humanidad se enfrenta a una enorme falta de agua. Afirma que, «a medida que el mundo se encamina hacia un periodo de escasez, el reto que se plantea a los gobiernos es

5 Su antecesor, el Acuerdo General sobre Aranceles Aduaneros y Comercio o GATT, sí tomó decisiones por mayoría en muy raras ocasiones. 


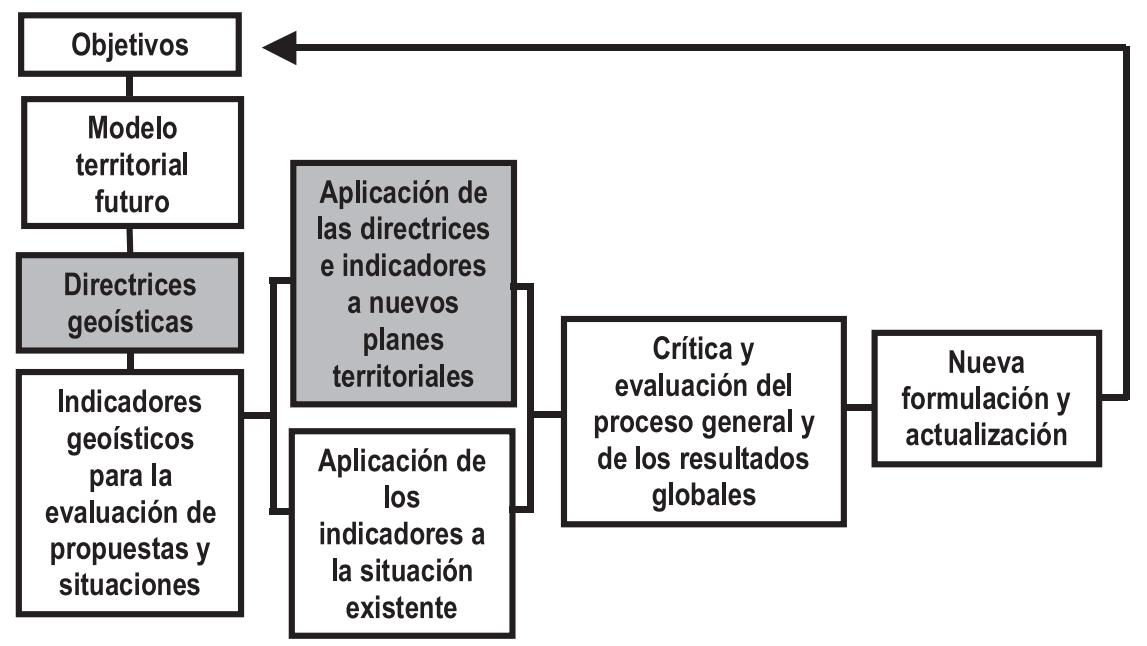

FiguRA 3. La planificación geoísta.

En gris se han resaltado las principales fases en que las propuestas se someten a la participación y opinión de expertos, administraciones, agentes sociales, asociaciones, etc.

adoptar una medida políticamente impopular: fijar precios del agua que reflejen su valor. El cobro del agua fomenta un consumo eficiente por parte de los consumidores». En la misma dirección apunta Lomborg ${ }^{6}$ (2003: 227 y 232), pero desde una perspectiva completamente diferente. Opina que «podemos disponer de agua suficiente siempre que podamos pagarla» y que «un precio más adecuado para el agua parece ser la cuestión principal a la hora de solucionar el problema... Asignar un precio al agua servirá para asegurar el mejor de los equilibrios posibles». Una directriz geoísta podría recomendar a las autoridades con responsabilidad en la materia el establecimiento de unas tarifas del agua que:

- Cubran el coste total del ciclo del agua ${ }^{7}$, desde la captación, conducción, potabilización, almacenamiento y distribución del agua potable, hasta el alcantarillado sanitario —recolección de aguas residuales, su tratamiento y, en el caso de los países con escasez de agua, su reutilización-, así como cualquier otro coste medioambiental asociado.

- Financien, al menos en parte, la conservación de los bosques ubicados en su área de captación de aguas, en tanto que éstos juegan un papel importante en la disponibilidad hídrica de la región al incrementar las filtraciones, disminuir el aterramiento de los embalses, contribuir a un aumento de la evaporación en la atmósfera, disminuir el riesgo de avenidas, etc.

- Financien, en los países desarrollados, parte de la conservación de los bosques de los países en vías de desarrollo, a través del fondo geoístico. Esto se justifica

6 Este autor ha sido fuertemente criticado por la falta de cientificidad en algunos de sus análisis, por ejemplo por Brockington (2003: 543-544), Gleick (2001:1-10), Pimm y Harvey (2001: 149-150), Rennie (2002:112), etc. Aun así, el conocer sus opiniones no deja de ser interesante, así como el contrastarlas con las de sus oponentes.

7 La Unión Europea ha avanzado en esta dirección al contemplarlo en el artículo 9 de la Directiva 2000/60/CE, de 23 de octubre de 2000, del Parlamento Europeo y del Consejo, que establece un marco comunitario de actuación en el ámbito de la política de aguas. 
porque las masas forestales contribuyen a disminuir el efecto invernadero, fundamentalmente debido a que «secuestran» en cierta medida el $\mathrm{CO}_{2}$ de la atmósfera mediante la fotosíntesis. La reducción en el aumento de las temperaturas que propiciaría el mantenimiento o la expansión de los bosques contribuiría a que hubiese una menor escasez de agua en numerosas regiones de la Tierra. Aunque el $\mathrm{CO}_{2}$ se vuelve a liberar de diversas maneras, por ejemplo con la caída de los árboles, la tala o los incendios, Pons (2004: 24) señala que la «actuación de los bosques como eficaces sumideros de carbono es muy importante para contribuir a la reducción de los cambios climáticos». Por ello, los proyectos de forestación y de reforestación son contemplados en el marco del Protocolo de Kioto como tipos de Mecanismo de Desarrollo Limpio (SGPCCC, 2004: 53), si bien se debería haber considerado también el mantenimiento de los bosques, porque, si no, incitan a que se talen y se vuelvan a replantar. La financiación de la conservación de los bosques aquí propuesta, que está relacionada con el agua, se completaría con otros fondos de mayor cuantía establecidos en función de su contribución a la biodiversidad y al no empeoramiento del calentamiento de la Tierra.

- Sean reducidas para el consumo doméstico básico indispensable, medido por conexión y, a ser posible, por persona, en aquellos países que se estime necesario, considerando al menos los sectores de la población o los barrios más marginales.

- Sean reducidas para la agricultura de las regiones poco desarrolladas, en las que una subida de precios haga inviable las explotaciones.

- Sean progresivas, aumentando por tramos de consumo conforme crece el gasto, si bien teniendo en cuenta factores relativos como el número de personas.

- Se implanten con una elevación inicial apreciable y un incremento progresivo a lo largo de los siguientes años, hasta cubrir todo el coste. Lógicamente, si los costes se redujesen por debajo de los ingresos recaudados, las tarifas disminuirían.

- Se implanten tras una campaña de participación pública y de información extensiva, que además de explicar las medidas fomenten el ahorro de agua.

- Se apliquen a todos los usos — agrarios, industriales y urbanos-.

- Tengan en cuenta la cultura y las estructuras sociales locales.

Estas medidas suelen ser impopulares y difíciles de aplicar, por lo que Dinar (2003: 34) recomienda que se implementen en el momento político adecuado, normalmente tras el comienzo de un mandato de gobierno. Rijsberman (2004: 520-521) señala que en cualquier momento dado, cerca de la mitad de la población de los países en desarrollo sufren una o más enfermedades relacionadas con el agua y que el saneamiento debe de recibir al menos la misma atención, y más financiación, que el suministro de agua potable.

Las directrices irían acompañadas por unos indicadores que permitiesen evaluar el grado de acercamiento a lo que postulan. En relación con la mencionada directriz de recuperación de los costes del agua, algunos ejemplo de indicadores podrían:

- Porcentaje que se recupera de los costes de los servicios asociados a los usos de agua.

- Porcentaje de personas que tienen una tarifa reducida para el consumo básico indispensable, respecto al total de la población y de las necesitadas.

- Incremento de la tarifa conforme aumentan los consumos, en términos relativos y absolutos.

- Variación en la evolución temporal de la tarifa, considerándose favorable el que se produzca un incremento al alza apreciable en poco tiempo, salvo que el punto de partida esté cerca o sea superior a la recuperación integral de costes. 
- Presupuesto dedicado a la participación de los agentes y a la campaña de información pública extensiva.

- Usos a los que se aplican tarifas que recuperan los costes del agua.

- Evolución del consumo de agua por persona.

- Eficiencia en el consumo del agua en relación con la actividad económica, medida por ejemplo en el agua consumida respecto al número de empleos de cada sector económico.

- Porcentaje de las aguas residuales que se depuran.

- Porcentaje de las aguas residuales que se reutilizan.

- Pérdidas de agua en los sistemas de distribución. Se considerarían valores desfavorables las pérdidas superiores al $25 \%$ en la red urbana, al $35 \%$ en las rurales y al $30 \%$ en redes intermedias, y favorables las pérdidas inferiores al 15\%, 25\% y $20 \%$ respectivamente (AEMA, 2002: 23).

Entre los indicadores posibles habrá que realizar una selección inicial que estará sometida a un proceso de continua revisión, incorporando nuevos y eliminando antiguos en función de su relevancia y de sus posibilidades de aplicación. Asimismo, cuando resulte justificado el sistema de indicadores habrá que adaptarlo a las características de cada área, no aplicando los que no resulten de interés y creando algunos específicos cuando sea necesario. Por ejemplo, la evolución en el consumo de agua por persona o la reutilización de las aguas residuales no adquiere el mismo valor en los lugares con abundantes precipitaciones que en los que tienen un gran déficit hídrico.

Las directrices deberían tender a ser integrales, es decir, intentar abarcar el conjunto de los aspectos territoriales, pero, puesto que esto sería demasiado amplio y complejo, se podría empezar por los elementos que pudiesen ser más relevantes, y que se pudiesen aprobar de forma independiente, como sucedería con la recuperación de los costes del agua. Serían adelantos parciales a un posible plan territorial más completo. Thornley y Rydin (2002: 9-10) han resaltado que en la era de la globalización el planeamiento tiene que encontrar «nuevas maneras de pensar sobre su papel y nuevas manera de funcionar». Particularmente, enfatizan que debe lograr respuestas más rápidas, combinando el corto con el largo plazo.

A continuación se expone otro ejemplo de posible directriz geoísta. Supóngase que un número suficiente de países acuerda aprobar unas directrices que propugnen la potenciación de redes policéntricas de ciudades que tengan una densidad razonablemente elevada, simultaneada con abundantes espacios verdes en el conjunto del territorio y en su interior. Los núcleos urbanos estarían estructurados externa e internamente en función del transporte público. La planificación territorial y urbana, como norma general, no permitiría nuevos desarrollos residenciales desvinculados de barrios relativamente densos, para los que estaría prevista una nueva estación de transporte público, preferiblemente de tren de cercanías, metropolitano o autobús de alta capacidad ${ }^{8}$. En aquellos municipios a los que no pueda llegar el transporte público de masas se permitirá un cierto crecimiento residencial, cuya cuantía se determinaría en función de la dinámica demográfica previsible. Cuando fuese conveniente, establecerían medidas adicionales para reducir la congestión, como un peaje urbano para acceder a la zona central del área metropolitana o, de menor efectividad hasta ahora, pero con cierto interés, el fomento del teletrabajo 9 . En toda la región se tendería a limitar las viviendas unifamiliares.

8 Como el de la ciudad brasileña de Curitiba (Ruano, 2002: 39; Montaner, 1999).

9 Ver: Saxena y Mokhtarian, 1997: 125 y 130 y Moktarian, 2001: 517-518. 
Esta directriz se justifica porque un conjunto de ciudades compactas, densas y con numerosos espacios verdes entre ellas y en su interior, tiene ventajas sociales, como un menor coste en la provisión de servicios y una mayor accesibilidad a los equipamientos. Asimismo, es una organización más recomendable, entre otras razones porque favorecen un menor consumo de agua y energía, así como una mayor utilización del transporte público. Por el contrario, las viviendas unifamiliares tienen un mayor coste medioambiental, fundamentalmente por su más alto consumo de agua, energía y suelo, así como por incentivar el transporte privado. Por otro lado, no parece sensato intentar concentrar todo el crecimiento en una única ciudad, sino en una red de ellas. Esto se debe a que las deseconomías de escala serían muy elevadas y a que el territorio se aprovecha mejor con una red de núcleos urbanos bien distribuidos.

En la aplicación de las directrices se contemplarían todas las excepciones que resultasen debidamente justificadas. Así, para una directriz que promueva ciudades con densidades relativamente elevadas, se podrían exceptuar las siguientes zonas: próximas a espacios de interés paisajístico o medioambiental; rurales; turísticas que pudiesen perder un porcentaje importante de sus clientes potenciales; carentes de capacidad constructiva; en las que el tipo de suelo o subsuelo no lo permita; etc.

Hay investigaciones que apuntan en contra de esta directriz. Deffis (2000: 104), sostiene que el modelo de ciudad ideal ha de tener como condicionante la baja densidad de vivienda. Otros autores presentan rangos más o menos amplios del número de viviendas por hectáreas que consideran aceptable. Por ejemplo, López (1999: 195) afirma que únicamente los conjuntos de vivienda con menos de 70 viviendas/hectárea alcanzan un grado de satisfacción suficiente para sus habitantes. Hildebrand (1999: 31) cita dos estudios que, desde el punto de vista de la sostenibilidad, consideran que pueden ser óptimas densidades de hasta 100 viviendas por hectárea ${ }^{10}$. Una tarea a realizar es concretar qué se entiende por una densidad razonablemente elevada.

No haría falta esperar a tener una estrategia geoística general aprobada para impulsar directrices territoriales mundiales que por sí mismas se considerasen positivas. Las directrices se podrían aplicar a escala planetaria, como podría ser una directriz referente a la protección de los espacios más valiosos del mundo, promoviendo a cambio, en los casos en que se estime necesario, programas de ayuda al desarrollo que fomenten iniciativas endógenas. Un primer paso lo constituye la elaboración de una clasificación mundial de los espacios a proteger, algo que realizó la Unión Internacional de Conservación de la Naturaleza - UICN - en 1978, con varias actualizaciones, «siendo valiosísima la aportación, hasta ahora insuperada en variedad y precisión de las categorías» (Crespo de Nogueira, 2002: 36). Asimismo, la UICN ha realizado un Sistema de Información Geográfico, a escala mundial (WDPA, 2005), que se puede consultar en la Red. Además de profundizar en las líneas abordadas por la UICN, convendría evaluar la gestión de los parques respecto a unos indicadores comunes e integrar la política de espacios naturales con otras, por ejemplo la mencionada de promover el desarrollo local a cambio de la conservación en los lugares en que se estime más conveniente. A este respecto, se debe tener en cuenta el siguiente consejo de Ojeda (2000: 285): «puede entenderse que es más fácil programar obras infraestructurales y adquisiciones patrimoniales que planificar cambios de mentalidad y procesos formativos, pero debe comprenderse también que el grado de desarrollo sostenible que sea capaz de alcanzar un territorio dependerá, fundamentalmente, de sus capacidades propias para crear

10 Ofrecen el dato en personas por hectárea. Para convertirlo se ha considerado una media de 3 personas por vivienda. 
un conjunto de actividades económicas productivas compatibles con la conservación y, a la vez, competitivas».

Las directrices geoísticas también se podrían aplicar por la planificación territorial, a escala supranacional, nacional y subnacional. Se podrían implementar en territorios de geometría variable y admitirían discontinuidades en los sistemas de planificación en cascada, es decir, se podrían aplicar a una comarca, aunque no se hubiese desarrollado antes en un ámbito territorial superior. La planificación supramunicipal utilizaría las directrices y los indicadores como referencia para hacer previsiones de mejora respecto a las que será contrastada. En el proceso de planificación - figura 4-, los indicadores se utilizarían: previamente, para evaluar el punto de partida; durante, para hacer una previsión de los efectos que generaría el propio plan; y, posteriormente, para evaluar los resultados. Los indicadores también se podrían utilizar para analizar la situación donde no haya planificación geoística, de forma que se pudiera comparar con los lugares que disponen de la misma.

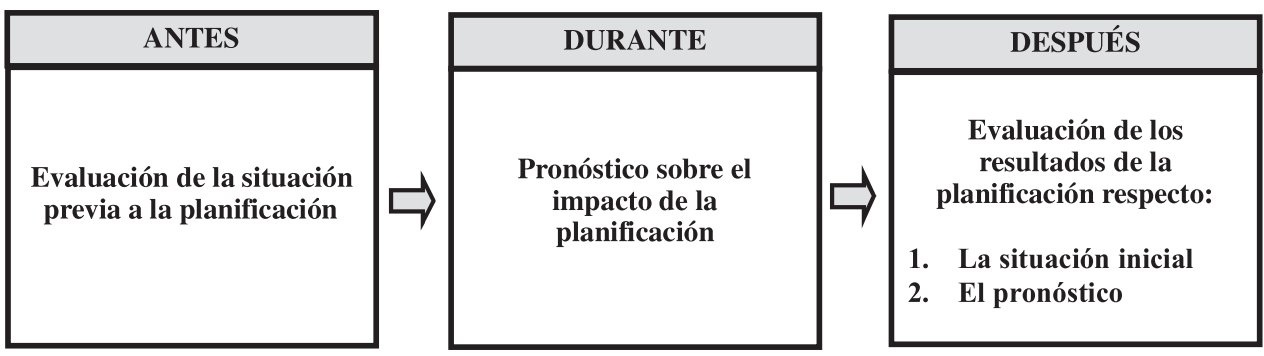

FIgURA 4. Aplicación de los indicadores geoísticos a la planificación supramunicipal.

Tras la aplicación de las directrices y de los indicadores geoístas en un cierto número de casos, se criticaría y evaluaría el proceso en su conjunto, para que se pueda aprender al máximo de los errores cometidos y se pueda difundir los aciertos logrados. Popper (1985: 154-155) resalta la importancia de la crítica para hacer avanzar el saber: «nuestro conocimiento aumenta mediante ensayo y eliminación de error» y «la principal diferencia entre su crecimiento precientífico y científico reside en que en el nivel científico buscamos conscientemente nuestros errores: la adopción consciente del método crítico resulta ser el principal instrumento del crecimiento»... «el método crítico, aunque debe usar contrastaciones siempre que sea posible, y preferiblemente las contrastaciones prácticas, puede ser generalizado en lo que yo describí como la actitud crítica o racional. Argumenté que uno de los mejores sentidos de «razón» y «razonabilidad» era la apertura a la crítica — disposición a ser criticado, y deseo de criticarse a sí mismo--; e intenté argüir que esta actitud crítica de razonabilidad debería ser extendida lo más lejos posible». La evaluación de la planificación territorial respecto a los indicadores geoísticos sería un tipo de contraste práctico, que habría que enmarcar en un proceso en el que se intenta ser todo lo autocrítico y crítico que sea factible, con el fin de llegar a mejores resultados.

La fase de evaluación llevaría a una nueva formulación y actualización de la estrategia territorial mundial. La planificación geoísta no es principalmente las directrices y los indicadores concretos de un momento dado, que lógicamente irán cambiando con el paso del tiempo, sino fundamentalmente el proceso permanente de elaborarlos. No es tanto la formulación relacionada con cada circunstancia particular, sino, sobre todo, el afán de superarse en el empeño de dar una respuesta eficaz de ordenación territorial planetaria, crecientemente necesaria ante el incremento imparable del fenómeno de la globalización. 